\title{
Inhaltsverzeichnis
}

16. Adjungierte Funktoren . . . . . . . . . . . . . . 1

16.1 Komposition von Funktoren und natürlichen Transformationen 1

16.2 Äquivalenzen von Kategorien . . . . . . . . . . . . . 2

16.3 Skelette .. . . . . . . . . . . . . . . . 5

16.4 Adjungierte Funktoren . . . . . . . . . . . . . . . . . . 8

16.5 Quasi-inverse Adjunktions-Transformationen . . . . . . . . 10

16.6 Völlig treue Adjungierte . . . . . . . . . . . . 15

16.7 Tensorprodukte . . . . . . . . . . . . . . . 19

17. Adjungierte Funktorpaare zwischen Funktorkategorien . . . . . . 22

17.1 Die Konstruktion von Kan . . . . . . . . . . . . . . . . 22

17.2 Dichte Funktoren . . . . . . . . . . . . . . . . . 29

17.3 Charakterisierung der Yoneda-Einbettung . . . . . . . 33

11.4 Kleine projektive Objekte . . . . . . . . . . . . . . . 36

17.5 Endlich erzeugte Objekte. . . . . . . . . . . . . . . . . 41

17.6 Natürliche Transformationen mit Parametern . . . . . . . . 43

17.7 Tensorprodukte über kleinen Kategorien . . . . . . . . . 45

17.8 Verwandte des Tensorprodukts . . . . . . . . . . . . . . 49

18. Grundzüge der Universellen Algebra . . . . . . . . . . . . . . 52

18.1 Algebraische Theorien . . . . . . . . . . . . . . 52

18.2 Yoneda-Einbettung und freie Algebren . . . . . . . . . . . 57

18.3 Unteralgebren und Covollständigkeit . . . . . . . . . . . . 61

18.4 Differenzcokerne und Kernpaare . . . . . . . . . . . . . 63

18.5 Algebraische Funktoren und Linksadjungierte. . . . . . . . 69

18.6 Semantik und Struktur. . . . . . . . . . . . . . . . . . 72

18.7 Kronecker-Produkt . . . . . . . . . . . . . . . . . . . 78

18.8 Charakterisierung algebraischer Kategorien . . . . . . . . . 81

19. Kalkül von Brüchen . . . . . . . . . . . . . . . . . . . 88

19.1 Kategorien von Brüchen . . . . . . . . . . . . . . . . . 88

19.2 Kalkül von Linksbrüchen . . . . . . . . . . . . . . . . . 89

19.3 Zerlegung von Funktoren und Saturation . . . . . . . . . . 94

19.4 Beziehungen zu Unterkategorien . . . . . . . . . . . . 100

19.5 Additivität und Exaktheit . . . . . . . . . . . . . . . . 104

19.6 Lokalisation in abelschen Kategorien. . . . . . . . . . . 109

19.7 Charakterisierung der Grothendieck-Kategorien mit Generator 115

20. Grothendieck-Topologien . . . . . . . . . . . . . . . . 121

20.1 Siebe und Topologien . . . . . . . . . . . . . . . . . 121

20.2 Bedeckende Morphismen und Garben. . . . . . . . . . 124 
$20.3 \mathrm{Zu}$ einer Prägarbe assoziierte Garbe . . . . . . . . . . . 128

20.4 Erzeugung von Topologien . . . . . . . . . . . . 137

20.5 Prätopologien. . . . . . . . . . . . . . . . . 139

Literatur . . . . . . . . . . . . . . . . . . 141

Sachverzeichnis zu Teil I und II . . . . . . . . . . . . . . 144

\section{Kategorien I}

\section{Inhaltsübersicht}

1. Kategorien

2. Funktoren

3. Kategorien von Kategorien und von Funktoren

4. Darstellbare Funktoren

5. Einige spezielle Objekte und Morphismen

6. Diagramme

7. Limites

8. Colimites

9. Filtrierende Colimites

10. Mengenwertige Funktoren

11. Objekte mit algebraischer Struktur

12. Abelsche Kategorien

13. Exakte Folgen

14. Colimites von Monomorphismen

15. Injektive Hüllen

Literatur

Sachverzeichnis zu Teil I 